\title{
Detection of small RNAs in Bordetella pertussis and identification of a novel repeated genetic element
}

\author{
David Hot ${ }^{1,2,3,4,5^{*}}$, Stéphanie Slupek ${ }^{1,2,3,4,5}$, Bérénice Wulbrecht1 ${ }^{1,2,3,4,5}$, Anthony D'Hondt ${ }^{1,2,3,4,5}$, Christine Hubans ${ }^{5,6}$, \\ Rudy Antoine ${ }^{1,2,3,4,5}$, Camille Locht ${ }^{1,2,3,4,5}$ and Yves Lemoine ${ }^{1,2,3,4,5}$
}

\begin{abstract}
Background: Small bacterial RNAs (sRNAs) have been shown to participate in the regulation of gene expression and have been identified in numerous prokaryotic species. Some of them are involved in the regulation of virulence in pathogenic bacteria. So far, little is known about sRNAs in Bordetella, and only very few sRNAs have been identified in the genome of Bordetella pertussis, the causative agent of whooping cough.

Results: An in silico approach was used to predict sRNAs genes in intergenic regions of the B. pertussis genome. The genome sequences of B. pertussis, Bordetella parapertussis, Bordetella bronchiseptica and Bordetella avium were compared using a Blast, and significant hits were analyzed using RNAz. Twenty-three candidate regions were obtained, including regions encoding the already documented 6S RNA, and the GCVT and FMN riboswitches. The existence of sRNAs was verified by Northern blot analyses, and transcripts were detected for 13 out of the 20 additional candidates. These new sRNAs were named Bordetella pertussis RNAs, bpr. The expression of 4 of them differed between the early, exponential and late growth phases, and one of them, bprJ2, was found to be under the control of BvgA/BvgS two-component regulatory system of Bordetella virulence. A phylogenetic study of the bprJ sequence revealed a novel, so far undocumented repeat of $~ 90 \mathrm{bp}$, found in numerous copies in the Bordetella genomes and in that of other Betaproteobacteria. This repeat exhibits certain features of mobile elements.
\end{abstract}

Conclusion: We shown here that B. pertussis, like other pathogens, expresses sRNAs, and that the expression of one of them is controlled by the BvgA/BvgS system, similarly to most virulence genes, suggesting that it is involved in virulence of $B$. pertussis.

\section{Background}

Small bacterial RNAs (sRNAs) have recently been shown to participate in the regulation of gene expression, and have been identified in numerous prokaryotic species [1-4]. They act mainly by antisense base pairing with their target mRNAs, often within a complex comprising the Sm-like RNA chaperone Hfq [5-7] or by direct binding to proteins resulting in the modulation of their activity $[8,9]$. Some sRNAs are involved in the regulation of virulence in several pathogenic bacteria [10-16]. These sRNAs function either directly on virulence genes or on their regulators. They act in parallel with protein regulatory systems in order to fine-tune the expression of

\footnotetext{
* Correspondence: david.hot@pasteur-lille.fr

'Center for Infection and Immunity of Lille (CIIL), Institut Pasteur de Lille, Lille, France

Full list of author information is available at the end of the article
}

virulence genes. For example, the Staphylococcus aureus RNAIII regulatory RNA is the effector molecule of the quorum sensing system arg, composed of a twocomponent system $(\operatorname{ArgA} / \mathrm{C})$ sensing a small autoinducing peptide, which binds and activates ArgC. This autoinducing peptide is processed from the propeptide (ArgD) by the peptidase (ArgB) [17].

In this work we focused on the pathogenic bacterium Bordetella pertussis, the causative agent of whooping cough, which remains an important global health problem, with up to 300,000 annual deaths and approximately 45 million cases each year $[18,19]$. Most deaths occur in young, unvaccinated children, but the incidence is also increasing in vaccinated populations, with regular epidemic outbreaks since the 1990 s [20-22]. Several causes have been attributed to this resurgence, including increased awareness and improved diagnostics of the
Ciomed Central

() 2011 Hot et al; licensee BioMed Central Ltd. This is an Open Access article distributed under the terms of the Creative Commons Attribution License (http://creativecommons.org/licenses/by/2.0), which permits unrestricted use, distribution, and reproduction in any medium, provided the original work is properly cited. 
disease, waning of vaccine-induced immunity and pathogen adaptation $[19,22]$.

A large proportion of Bordetella genes undergo a change in their expression during the infectious cycle [23-25] some of which correspond to phenotypic modulation under the control of a two-component system named BvgA/BvgS (reviewed in [26]). In this system BvgS acts as a sensor anchored in the inner membrane and BvgA as the transcriptional activator $[27,28]$. So far, little is known about sRNAs in Bordetella and their potential role in virulence or adaptation. Only three Bordetella sRNAs have been identified, the 6S RNA [29], the tmRNA [30] and a sRNA discovered by serendipity and lying in the opposite direction of the $b v g A / S$ mRNA $5^{\prime}$ untranslated region (UTR) [31,32]. The aim of this study was to scan the $B$. pertussis genome in order to identify potential sRNAs and to investigate whether some of them might be related to bacterial virulence. We used a general bioinformatics approach [33] and predicted 20 locations putatively bearing sRNA genes in intergenic regions (IGR) of the $B$. pertussis genome. Transcription was confirmed by Northern blot analyses for 13 of these locations. We further studied the expression of these new sRNAs under phenotypic modulation and showed that one of them is under the control of the BvgA/BvgS system, suggesting its role in virulence.

\section{Methods}

\section{Bacterial strains and growth conditions}

The genome sequences of $B$. pertussis TohamaI, Bordetella parapertussis 12822, Bordetella bronchesipteca RB50 [34] and Bordetella avium N197 [35] were used in the computational analyses.

B. pertussis BPSM, a streptomycin-resistant derivative of TohamaI [36], and BPLOW, an avirulent $b v g A S$ deletion mutant [37], were grown as previously described [24] at $37^{\circ} \mathrm{C}$ in modified Stainer-Scholte medium, containing $100 \mu \mathrm{g} / \mathrm{ml}$ streptomycin (Sigma Chemicals). Cultures were stopped either at early $\left(\mathrm{OD}_{600 \mathrm{~nm}} \sim 0.9\right)$, exponential $\left(\mathrm{OD}_{600 \mathrm{~nm}} \sim 1.8\right)$ or stationary phase $\left(\mathrm{OD}_{600 \mathrm{~nm}} \sim 3.8\right)$ by adding $2 \mathrm{ml}$ of 5:95 (v:v) phenol/ethanol to $8 \mathrm{ml}$ of culture medium. After centrifugation for $8 \mathrm{~min}$. at $2800 \times g$ the pellets were stored at $-80^{\circ} \mathrm{C}$ until further use.

For phenotypic modulation a 630-ml culture was grown in the same conditions until an $\mathrm{OD}_{600 \mathrm{~nm}}$ of approximately 0.7. The culture was then split into three, and phenotypic modulation was induced by adding $40 \mathrm{ml}$ of a pre-warmed $125 \mathrm{mM}$ nicotinic acid (20 mM final concentration) or $40 \mathrm{ml}$ of pre-warmed $300 \mathrm{mM} \mathrm{MgSO}_{4}$ (48 mM final concentration) in the two first sub-cultures. The third subculture served as control and received $40 \mathrm{ml}$ of sterile water pre-warmed at $37^{\circ} \mathrm{C}$. Culturing was then continued at $37^{\circ} \mathrm{C}$, and samples were taken for RNA extraction, just before induction $(\mathrm{t}=0)$ and at $\mathrm{t}=1 \mathrm{~min} ., 10 \mathrm{~min} ., 1 \mathrm{~h}$, $2 \mathrm{~h}, 6 \mathrm{~h}, 10 \mathrm{~h}$ and $30 \mathrm{~h}$ post-induction.

\section{In silico search of sRNAs}

The bacterial genome sequences and annotation files were obtained from the NCBI databases [38] and from the Sanger Institute [39]. Gene coordinates were used to extract IGRs using a parser in Perl. Homologous regions of these IGR sequences were searched in different bacterial genomes using local BLAST. Hits presenting more than $60 \%$ sequence identity over at least 70 nucleotides were analyzed further. A multiple alignment of these homologous regions was then obtained using ClustalW. These alignments were used to evaluate secondary structure conservation and thermodynamic stability using the software "RNAz" developed by Washietl et al. $[33,40]$.

\section{RNA extraction and Northern blot analyses}

The bacterial pellets were resuspended in $200 \mu \mathrm{l}$ of $1 \mathrm{mg} / \mathrm{ml}$ Lysozyme (Sigma Aldrisch). Total RNA was then extracted using the TRI Reagent kit (Ambion), following the recommendations of the supplier. The RNA quality was checked using Bioanalyzer 2100 (Agilent Technologies) before denaturation and electrophoresis $(10 \mu \mathrm{g}$ per lane) on a $10 \%$ acrylamide:bis-acrylamide (37.5:1) denaturing gel in $0.5 \times$ TBE buffer in the presence of $8 \mathrm{M}$ urea. The RNA was then transferred onto BrightStar Plus (Ambion) nylon membranes and UV-crosslinked. Biotinylated oligonucleotides (see Additional file 1, table S1), designed using FastPCR [41], were then used for hybridization in Northern Max buffer (Ambion), and the blots were developed by chemiluminescent detection using the BrightStar Biodetect kit (Ambion).

\section{Local DNA alignment}

Homology searches were performed with the program YASS [42] in local using the default parameters.

For the similarity search of the bprJ1 sequence, the program YASS was used on the web server [43] using the default parameters, except for the 'Hit criterion strategy', which was set on 'double hit'. The sequence of the IGR containing bprJ1 (between positions 3605200 and 3605405) was searched for homology in the $B$. pertussis genome. The homologous regions were then aligned using ClustalW, and the sequence closest to the consensus sequence (i.e. the sequence from position 2208359 to 2208448) was used as a query for the search in all bacterial genome sequences.

For the global sequence similarity search, the $B$. pertussis IGR sequences longer than 140 nucleotides and not containing tRNA or rRNA genes were compared using YASS with all complete bacterial genome sequences available in GenBank, except those of the 
Bordetella genus. Hits with a score $>50$ and with $>70 \%$ identity were considered further.

\section{Results and discussion}

In silico prediction of sRNAs and validation by Northern blot analysis

Except for the 6S RNA [29], the tmRNA [30] and a small RNA transcribed in antisense to the $b v g A$ mRNA [31,32], no sRNA has been identified so far in Bordetella. We used the 'RNAz' algorithm [33], which is based on a search for structure conservation between closely related bacterial genomes. Predictions using different combinations of betaproteobacteria genomes resulted in various numbers of candidates. We choose to work on a prediction obtained comparing genomes of B. pertussis Tohama I, B. bronchiseprica RB50, B. parapertussis 12822 and B. avium 197N. This search resulted in the prediction of 23 sequences for potential sRNAs in the $B$. pertussis genome (see Additional file 1, table $\mathrm{S} 2$ for the detailed list of predictions). The same search strategy using only $B$. pertussis Tohama I, B. bronchiseprica RB50 and B. parapertussis 12822 resulted in 657 predictions. Among the 23 predicted sequences, the position of the 6S RNA gene was correctly designated (predicted coordinates from 3246822 to 3246972 , real coordinates from 3246812 to 3246993 ), as well as the position of 2 riboswitches already described in the Rfam database [44]. The first, GCVT, is selectively triggered by glycine [45] and controls the translation of the aminomethyltransferase mRNA (BP0195). The second riboswitch belongs to the flavin mononucleotide (FMN) class [46] and regulates the translation of ribB (BP0471).

\section{Validation by Northern blot analysis}

All 23 predicted regions, except the ones of the GCVT riboswitch, the FMN riboswitch and the $6 \mathrm{~S}$ RNA, were tested for transcription of small-size RNA by Northern blot analyses. Biotinylated oligonucleotide probes were designed for both strands of the 20 predicted regions (see probe sequences in Additional file 1, table S1) and used to test the presence and orientation of short transcripts in Northern blot analyses. As a positive control, an additional probe was designed that corresponds to the sRNA characterized by Scarlato et al. [31,32]. When total RNA extracted from B. pertussis cultures stopped at an $\mathrm{ODs}_{600}$ $\mathrm{nm}$ of $0.9,1.8$ or 3.6 was analyzed, 13 positions in addition to the positive control showed evidence of transcription (see Additional file 1, figure S1). The transcription profile of these 14 genomic regions was further analyzed for the kinetics of expression over $30 \mathrm{~h}$ of culture [from $\mathrm{OD}_{600 \mathrm{~nm}}$ $\sim 0.7$ to $\sim 7.9$ ] (Figure 1 ). All the bands clearly detected in the Northern blots in the validation experiments (Figure 1 and Additional file 1, figure S1) and in the phenotypic modulation experiments (see below) were considered as transcripts. Each detected transcript was labeled with a capital letter. A number was added after the letter to discriminate transcripts in case of multiple bands (e.g. TranscriptA1, TranscriptA2). Locations for which transcription was detected on both strands were distinguished with an apostrophe, e.g. TranscriptM, TranscriptM' (Figure 1 and Table 1).

The analysis of five genomic positions resulted in the detection of 2 bands (transcripts A1 and A2, D1 and D3, E1 and E2, L1 and L2, N1 and N2), indicating the presence of two overlapping transcripts of different lengths, as has been seen for several sRNAs of Pseudomonas aeroginosa [47] and for Escherichia coli IstR1 and IstR2, which have each a specific function and are generated from separate promoters but share a common 5 end [48]. Alternatively, these bands could be the result of a post-transcriptional processing of a single RNA transcript [47].

Some transcripts were only produced during the exponential phase (transcripts A1, A2 and F). Transcript D1 was expressed at higher levels during exponential phase compared to the early and stationary phases, whereas Transcript D2 did not show any significant modification of expression during growth. This observation rules out the possibility that Transcript D1 is the precursor of Transcript D2, and argues that the two transcripts are independent and under the control of different factors. In contrast, Transcripts A1, A2 and Transcripts F1, F2, are expressed at similar levels during the different growth phases. They may therefore each be produced from a unique RNA and subsequently processed into a shorter version. Finally, two regions gave rise to a transcript from both strands. Transcripts L1 and L' are transcribed respectively from the lagging and leading strand template of predicted region 3811548-3811699. Transcripts $M$ and $M^{\prime}$ are transcribed respectively from the lagging and leading strand template of predicted region 3896371-3896457. In both cases, the two transcripts had the same apparent size ( $\sim 80$ nucleotides for transcripts L1 and L' and 190 nucleotides for transcripts M and M'). For these two pairs of transcripts, the biotinylated probes used to detect transcription on both strands were designed approximately at the same coordinates, indicating that the observed transcripts are complimentary to each other for most of their lengths (Figures 1 and $2 \mathrm{~B}$ ).

Most regulatory sRNAs are synthesized as discrete transcripts under the control of dedicated promoter and terminator sequences. However, some bacterial regulatory elements correspond to sequences at the $5^{\prime}$ or 3' UTRs of mRNAs that can adopt different conformations 


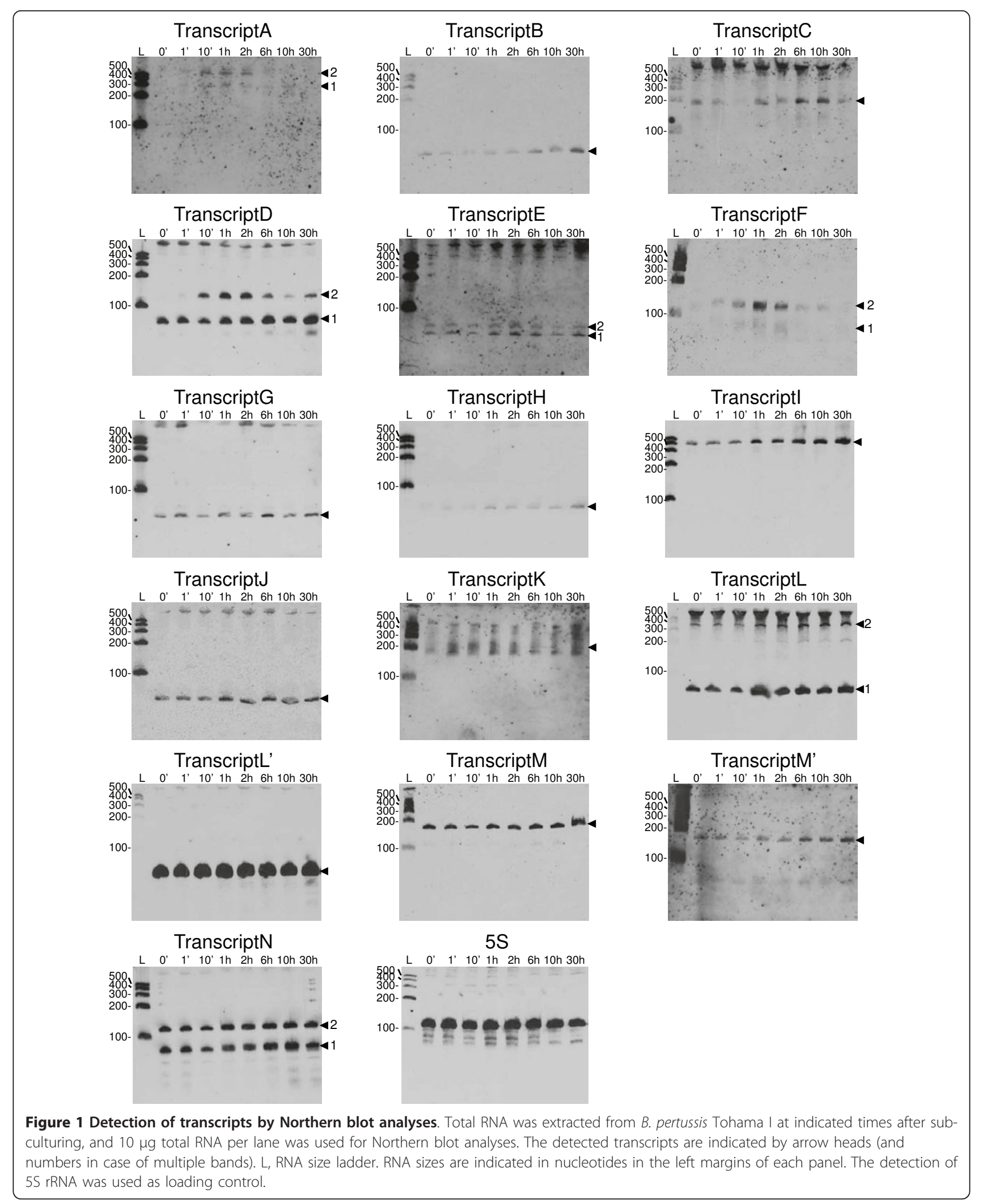


Table 1 Summary of identified sRNA features

\begin{tabular}{|c|c|c|c|c|c|c|c|c|c|}
\hline $\begin{array}{l}\text { Transcript } \\
\text { name }\end{array}$ & $\begin{array}{l}\text { Gene } \\
\text { name }\end{array}$ & $\begin{array}{l}\text { Predicted } 5^{\prime} \\
\text { coordinate }^{a}\end{array}$ & $\begin{array}{l}\text { Predicted 3' } \\
\text { coordinate }^{a}\end{array}$ & $\begin{array}{l}\text { Predicted } \\
\text { length }^{b}\end{array}$ & $\begin{array}{c}\text { Approx. } \\
\text { obs. length }\end{array}$ & $\begin{array}{c}\text { SRNA } \\
\text { orientation }^{d}\end{array}$ & $\begin{array}{c}\text { IGR } \\
\text { length }\end{array}$ & $\begin{array}{c}5^{\prime} \text { gene name and } \\
\text { orientation }^{f}\end{array}$ & $\begin{array}{c}3^{\prime} \text { gene name and } \\
\text { orientation }^{f}\end{array}$ \\
\hline TranscriptA1 & bprA1 & 488504 & 488654 & 150 & 250 & $<$ & 522 & BP0475 < & BP0477 > \\
\hline TranscriptA2 & bprA2 & 488504 & 488654 & 150 & 300 & $<$ & 522 & BP0475 < & BP0477 > \\
\hline TranscriptB & bprB & 1494130 & 1494207 & 77 & 80 & $>$ & 726 & BP1418< & BP1419 > \\
\hline TranscriptC & bprC & 1968374 & 1968529 & 155 & 190 & $>$ & 425 & BP1878< & BP1879 > \\
\hline TranscriptD1 & bprD1 & 2624007 & 2624157 & 150 & 90 & $<$ & 783 & BP2479 < & BP2480 > \\
\hline TranscriptD2 & bprD2 & 2624007 & 2624157 & 150 & 110 & $<$ & 783 & BP2479 < & BP2480 > \\
\hline TranscriptE1 & bprE1 & 2699394 & 2699487 & 93 & 70 & $>$ & 239 & BP2546 < & BP2547 > \\
\hline TranscriptE2 & bprE2 & 2699394 & 2699487 & 93 & 80 & $>$ & 239 & BP2546 < & BP2547 > \\
\hline TranscriptF1 & bprF1 & 3099570 & 3099720 & 150 & 80 & $<$ & 256 & BP2908 < & BP2909 < \\
\hline TranscriptF2 & bprF2 & 3099570 & 3099720 & 150 & 150 & $<$ & 256 & BP2908 < & BP2909 < \\
\hline TranscriptG & bprG & 3173584 & 3173734 & 150 & 70 & $<$ & 305 & BP2982 > & BP2983 > \\
\hline TranscriptH & bprH & 3178090 & 3178331 & 241 & 70 & $>$ & 318 & BP2984 > & BP2985 > \\
\hline Transcriptl & bprl & 3263729 & 3263815 & 86 & 450 & $<$ & 277 & BP3061 > & BP3062 > \\
\hline TranscriptJ & bprJ & 3605317 & 3605397 & 80 & 70 & $>$ & 206 & BP3395 < & BP3396 < \\
\hline TranscriptK & bprK & 3619230 & 3619365 & 135 & 200 & $<$ & 316 & BP3410 < & BP3411 > \\
\hline TranscriptL1 & bprL1 & 3811548 & 3811699 & 151 & 80 & $<$ & 336 & BP3594 < & BP3595 > \\
\hline TranscriptL2 & bprL2 & 3811548 & 3811699 & 151 & 350 & $<$ & 336 & BP3594 < & BP3595 > \\
\hline TranscriptL' & bprL' & 3811548 & 3811699 & 151 & 80 & $>$ & 336 & BP3594 < & BP3595 > \\
\hline TranscriptM & bprM & 3896371 & 3896457 & 86 & 190 & $<$ & 281 & BP3686 > & BP3687 > \\
\hline TranscriptM' & bprM' & 3896371 & 3896457 & 86 & 190 & $>$ & 281 & BP3686 > & BP3687 > \\
\hline TranscriptN1 & bprN1 & 3956837 & 3956934 & 97 & 80 & $<$ & 393 & BP3747 > & BP3748 < \\
\hline TranscriptN2 & bprN2 & 3956837 & 3956934 & 97 & 110 & $<$ & 393 & BP3747 > & BP3748 < \\
\hline
\end{tabular}

${ }^{\mathrm{a} G e n o m i c ~ c o o r d i n a t e s ~ a s ~ p r e d i c t e d ~ b y ~ R N A z . ~}$

${ }^{b}$ In nucleotides.

'sRNA length, in nucleotides, determined from apparent sizes on Northern blots.

${ }^{d}$ Genomic orientation of sRNA transcripts. '>' = on positive strand: the strand given in the GenBank genome database (NC_002929); '<' = on negative strand: the complementary strand.

eLength, in nucleotides, of the IGRs containing sRNA genes (according to the annotation).

fGenomic orientation of flanking mRNAs. '>' = on positive strand: the strand given in the GenBank genome database (NC_002929); '<' = on negative strand: the complementary strand.

in response to environmental signals. This is the case for autoregulatory motifs and riboswitches [49]. Some of these regulatory elements can, under specific conditions, cause transcriptional arrest (reviewed in [50]). In order to test whether some of the transcripts detected in this study are abortive, processed or degraded forms of the 5'UTR or 3'UTR of their neighboring genes, a control RT-PCR was carried out on transcripts which were in close vicinity of and in the same orientation as their neighboring 5' or 3' ORF (see Additional file 2, figure S2 and table S3). All detected transcripts were found to result from independent transcription, except for Transcript $B$, which was linked to the transcription of the downstream gene rpsB (BP1419), encoding the 30S ribosomal protein S2. The function of S2 is still uncertain. It may potentially act as a bridge between the $16 \mathrm{~S}$ RNA and ribosomal protein $\mathrm{S} 1$ [51]. The ORF of $\operatorname{rps} B$ genes have recently been shown to be preceded by a conserved specific motif in the 5'UTR, which could be a cis- regulatory element binding to the S2 protein for an autoregulatory control of its own synthesis [52]. The existence of Transcript B suggests that in $B$. pertussis the 5'UTR of $\operatorname{rps} B$ contains a cis-regulatory element inhibiting the full-length transcription of the mRNA, as it has been demonstrated for other ribosomal protein genes $[53,54]$.

Finally, the coding potential of all transcripts was evaluated by a search for potential coding sequences in their genomic regions using the program 'ORF Finder' of the NCBI [55] (data not shown). As the B. pertussis genome appears to be under-annotated when the EasyGene prediction is compared to the RefSeq annotation [56], we also looked for predicted coding sequences or neighboring genes with alternative start codons in these genomic regions, using the prokaryotic gene-finder algorithm EasyGene. No coding sequences were predicted in the transcribed regions, even for the longest ones. 


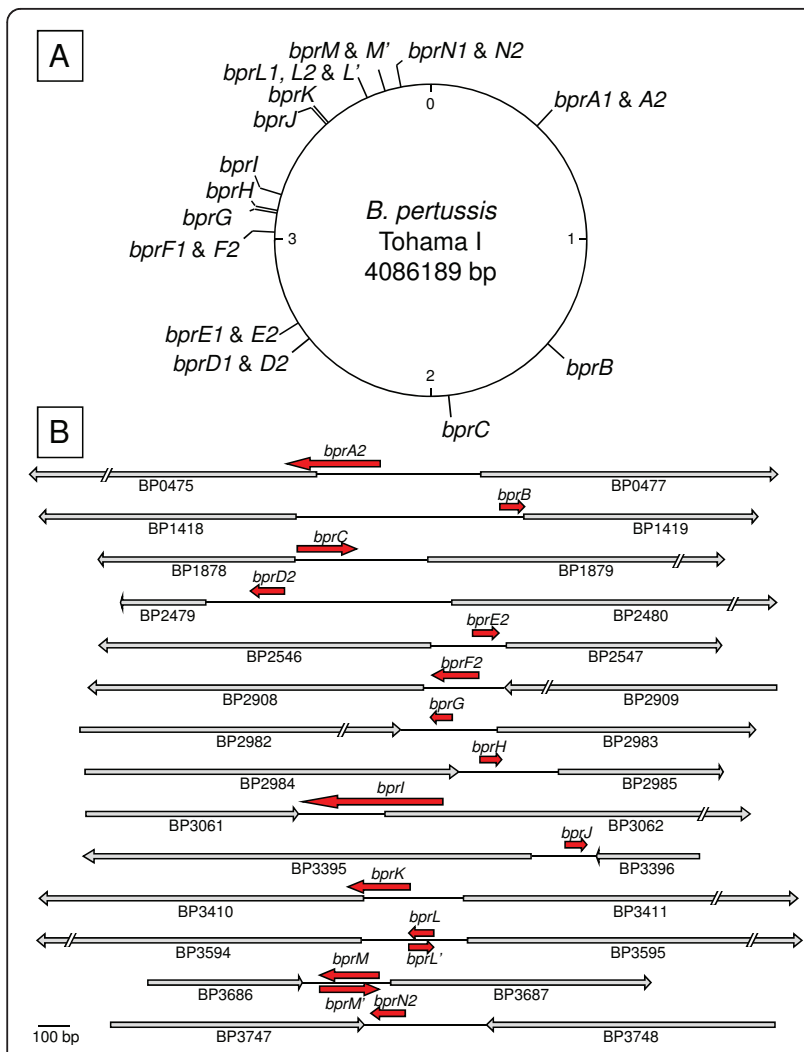

Figure 2 Chromosomal position and genomic context of the bpr loci of $\boldsymbol{B}$. pertussis. The positions of the $14 \mathrm{bpr}$ loci are shown on the chromosomal map of the $B$. pertussis genome. The numbers give an approximate chromosome graduation in Mbp (A). The positions and orientations of the bpr loci in the B. pertussis genome are depicted by the red arrows (B). Grey arrows represent the ORF of the bpr-adjacent genes and their orientations according to the GenBank annotation. The estimated positions of sRNAs were determined considering the positions of the biotynilated probes (see Additional file 1, table S2) and the apparent lengths of the transcripts on the Northern blots. In case of multi-band detection only the longest transcript is represented.

In view of these observations we conclude that all detected transcripts are genuine sRNAs and were therefore named Bordetella pertussis RNAs (BprA1, A2, B, C, ...). The rpsB gene probably contains in its 5'UTR an autoregulatory element, and $\mathrm{BprB}$ most likely results from the inhibition of transcription of the rpsB mRNA. The validated sRNA genes are scattered throughout the B. pertussis chromosome (Figure 2A). The location of these sRNA genes relatively to their neighboring genes was determined by the biotinylated probe coordinates and the apparent sizes on the Northern blots (Figure 2B).

\section{Features of the B. pertussis sRNAs}

Specific and general features of the B. pertussis sRNAs were further analyzed and compared with those of other bacterial sRNAs. The genes of the B. pertussis sRNAs are located in IGR of various lengths, comprised between $206 \mathrm{bp}$ and $783 \mathrm{bp}$. They have no apparent preference for lagging or leading strand templates, but they are preferentially located on one of the two replichores (79\%) (See Figure 2A). These characteristics are similar to those of the E. coli sRNA genes [57].

Some of the B. pertussis sRNA genes (BprC, I and N) are very close to or overlap adjacent ORFs in the opposite orientation and thus are likely to act on their mRNA, whereas others are more distant from the adjacent ORFs and/or in the same orientation (See Figure $2 \mathrm{~B})$. The potential mRNA targets of all sRNAs were predicted using TargetRNA algorithm [58]. The predicted gene targets were compared to documented targets of sRNAs from other bacteria. However, none of the

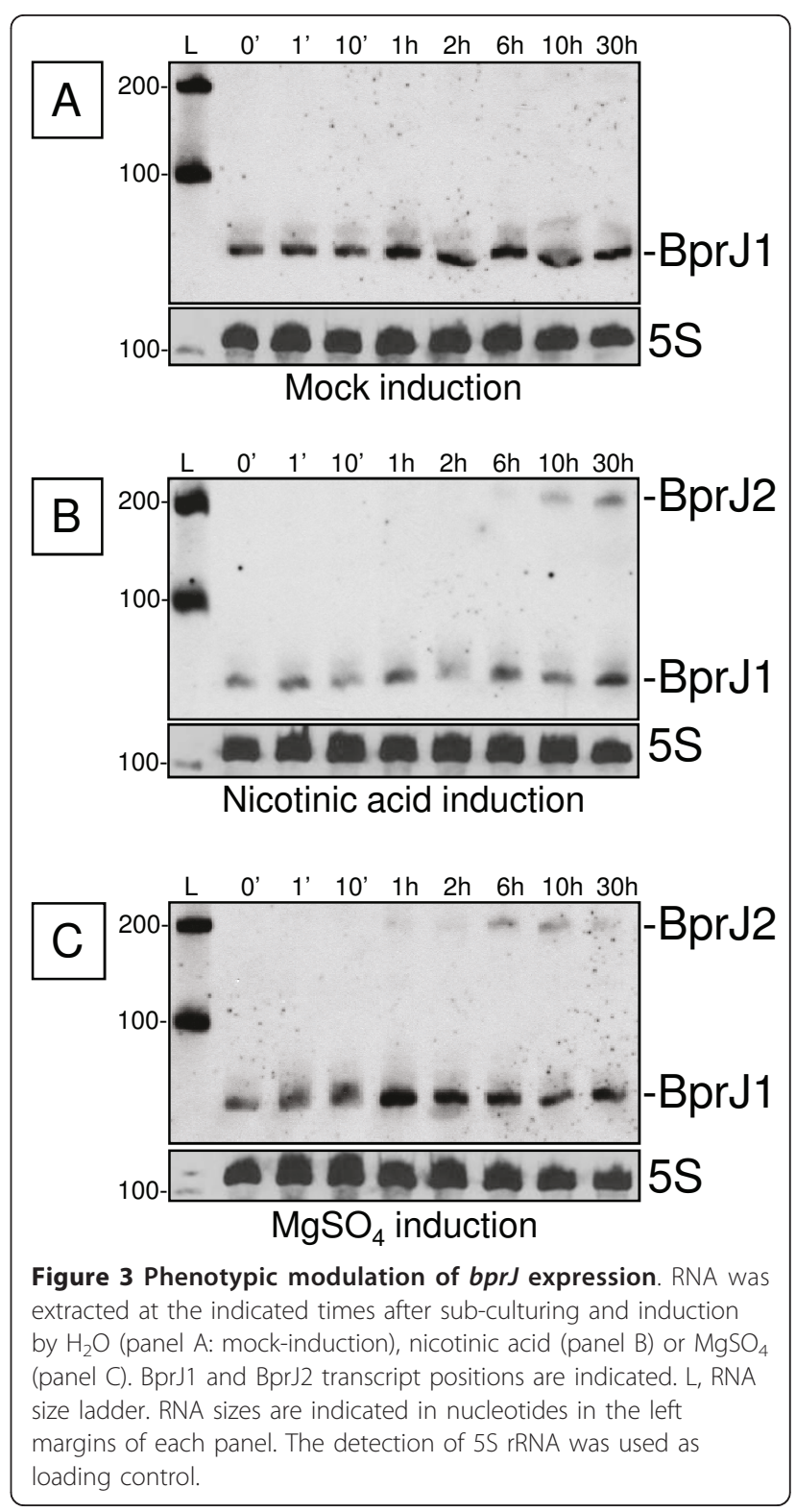


predicted $B$. pertussis mRNA targets was orthologous to a sRNA target from another bacterium (data not shown). Similarly, orthologous genes of the B. pertussis sRNA-flanking genes were searched in the Rfam and Noncode databases, as some sRNAs are cis-encoded antisense or cis-acting elements. The only product mentioned in these databases is the ribonuclease $\mathrm{E}$ gene (ortholog to BP0475), which flanks bprA and is in the same orientation. BprA might therefore be the 5'UTR cis-acting element of the ribonuclease $\mathrm{E}$ gene (rne 5'UTR), which codes for a key enzyme in the mRNA degradation pathway, including its own. This rne 5'UTR element acts as a sensor of the cellular RNase E concentration and allows for auto-regulation of its mRNA degradation [59].

\section{Phenotypic modulation of B. pertussis sRNAs}

To investigate whether some of the new sRNAs genes may potentially be under the control of the two-component system BvgA/S and therefore potentially be

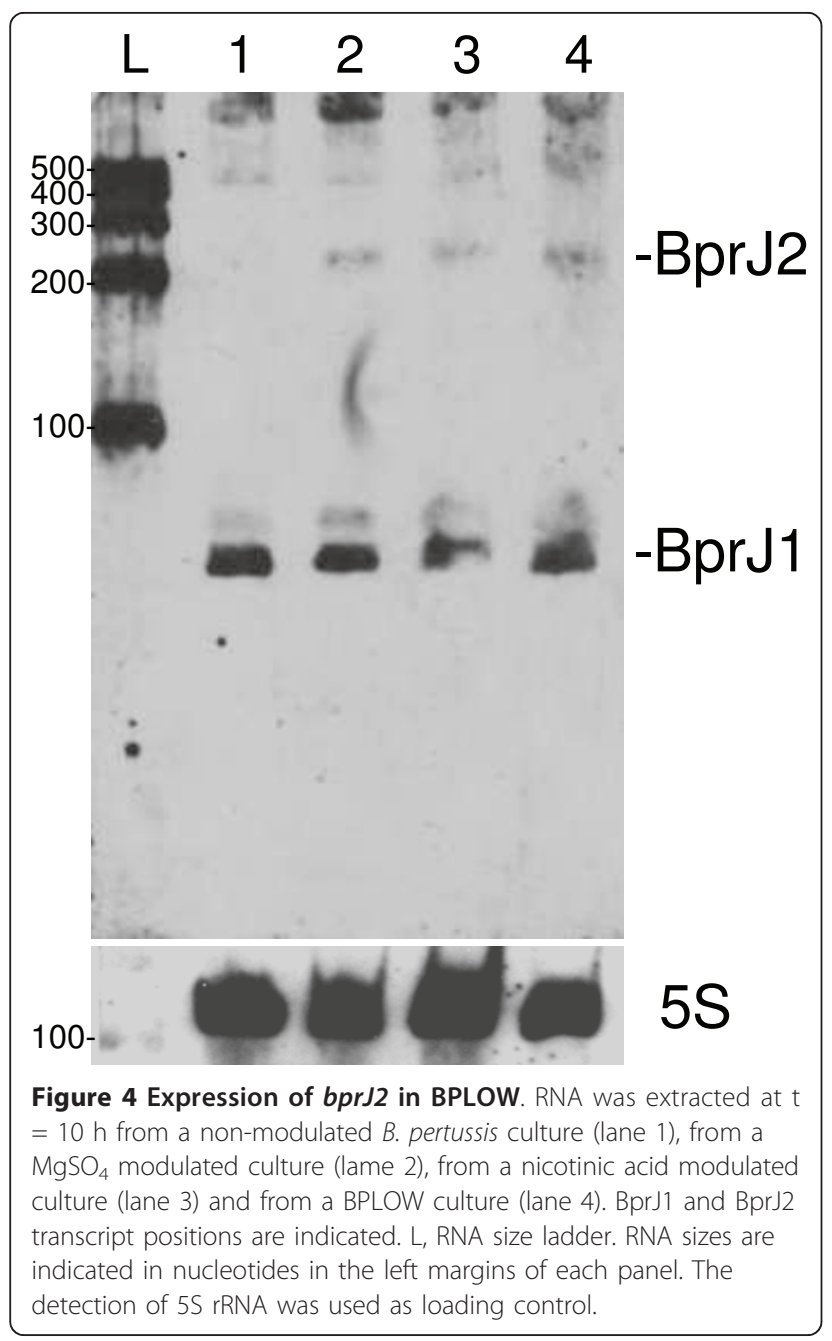

involved in Bordetella virulence regulation, phenotypic modulation was induced by adding nicotinic acid or $\mathrm{MgSO}_{4}$ in the growth medium in order to switch the bacteria from the virulent $\left(\mathrm{Bvg}^{+}\right.$phase) to the non-virulent phase (Bvg- phase) $[60,61]$. The modulation of expression was monitored by Northern blot analyses for up to $30 \mathrm{~h}$ after induction (data not shown) and the expression profiles were compared to those of the noninduced culture. One sRNA, BprJ, showed a change in its expression compared to the control culture. The 80nucleotides band detected in the non-modulated culture did not change after phenotypic modulation, but a second band of approximately 200 nucleotides appeared 6 $\mathrm{h}$ and $10 \mathrm{~h}$ after induction of phenotypic modulation by $\mathrm{MgSO}_{4}$ and nicotinic acid, respectively (Figure 3). This new band is indicative of longer transcript overlapping the 80-nucleotide transcript and appears to be specific for the non-virulent phase ( $\mathrm{Bvg}^{-}$phase). According to the nomenclature adopted above, this second transcript was named BprJ2 to distinguish it from the 80-nucleotides transcript, which was named BprJ1.

To confirm that this new transcript is under the control of the BvgA/BvgS system, its expression was monitored by Northern blot analysis in the avirulent BPLOW mutant in which part of the $b v g A-b v g S$ operon had been deleted [37]. BprJ2 was also detected in the BPLOW RNA (Figure 4), confirming its $v r g$ (virulence repressed gene) feature. The other vrgs of $B$. pertussis mainly encode cell-envelope, small-molecule degradation or hypothetical proteins $[23,24]$ and include the previously

\section{Table 2 Number of BRE detected in different bacterial} strains

\begin{tabular}{|c|c|c|c|}
\hline $\begin{array}{l}\text { Nbr. of } \\
\text { repeats }\end{array}$ & Strain & Familly & Acc.number \\
\hline 8 & Bordetella avium 197N & Alcaligenaceae & NC_001064 \\
\hline 22 & $\begin{array}{l}\text { Bordetella bronchiseptica } \\
\text { RB50 }\end{array}$ & Alcaligenaceae & NC_002927 \\
\hline 10 & $\begin{array}{l}\text { Bordetella parapertussis } \\
18822\end{array}$ & Alcaligenaceae & NC_002928 \\
\hline 8 & $\begin{array}{l}\text { Bordetella pertussis } \\
\text { Tohama I }\end{array}$ & Alcaligenaceae & NC_002929 \\
\hline 1 & Azoarcus sp. EbN1 & Rhodocyclaceae & NC_006513.1 \\
\hline 4 & $\begin{array}{l}\text { Rhodoferax ferrireducens } \\
\text { T118 }\end{array}$ & Comamonadaceae & NC_007908.1 \\
\hline 29 & Polaromonas sp. JS666 & Comamonadaceae & NC_007948.1 \\
\hline 4 & $\begin{array}{l}\text { Acidovorax avenae subsp. } \\
\text { citrulli AAC00-1 }\end{array}$ & Comamonadaceae & NC_008752.1 \\
\hline 4 & $\begin{array}{l}\text { Polaromonas } \\
\text { naphthalenivorans CJ2 }\end{array}$ & Comamonadaceae & NC_008781.1 \\
\hline 1 & Acidovorax sp. JS42 & Comamonadaceae & NC_008782.1 \\
\hline 1 & $\begin{array}{l}\text { Verminephrobacter } \\
\text { eiseniae EF01-2 }\end{array}$ & Comamonadaceae & NC_008786.1 \\
\hline 13 & Delftia acidovorans SPH-1 & Comamonadaceae & NC_010002.1 \\
\hline
\end{tabular}


described but uncharacterized $\operatorname{vrg6}, \operatorname{vrg} 18, \operatorname{vrg} 24$ and $\operatorname{vrg73}$ genes [62].

\section{Identification of a novel Betaproteobacteria repeated element}

A local DNA sequence analysis using YASS showed that the bprJ1 and bprJ2 genomic region contains a sequence, from coordinates 3605317 to 3605401 , highly repeated in the Bordetella (see Additional file 1, list S1) and in several other Beta-proteobacteria genomes (Table 2). We named it therefore Beta-proteobacterial repeat element (BRE). This repeated element is found as a fulllength sequence of $\sim 90 \mathrm{bp}$ or as partial but conserved sequence (between $\sim 60$ and $\sim 40 \mathrm{bp}$ ) with a maximum number of 29 repeats (with scores $>50$ and $>70 \%$ identity) in the genome of Polaromonas sp. JS666 (see Additional file 1, table S4). All identified repeated elements were located in chromosomal DNA, usually in IGRs, although some overlap ORFs at their 5' or 3' ends by a few bases. Surprisingly, some of the repeats are located entirely within coding sequences.

Some IGRs and ORFs contain more than one BRE (a maximum of 8 repeats was observed in B. bronchiseptica between BB2301 (sphB3) and BB3202) organized in tandem with no or only a few nucleotides between each repeat. The complete and partial BRE sequences are scattered around the chromosomes of $B$. pertussis, $B$. bronchiseptica, B. parapertussis and B. avium (Figure 5). An analysis of the Bordetella BRE sequences revealed 44 full-length repeats, ranging from 75 to $91 \mathrm{bp}$ in length, and 1 partial repeat of $50 \mathrm{bp}$. They exhibit an average GC-content of $69.0 \%$ (ranging from $63.7 \%$ to $78.9 \%$ ), which is higher than the average GC-content of IGR sequences $(62.9 \%$ for IGRs $\geq 10 \mathrm{bp})$ and slightly higher than the average $\mathrm{GC}$-content of the complete genome sequences $(67.7 \%)$. The orientations of the repeats, inferred by the transcriptional orientation of bprJ1 and bprJ2, are not co-orientated with the direction of the replication fork.

The genetic context, i.e. the nature and orientation of the BRE flanking genes, is only moderately conserved between B. pertussis, B. bronchiseptica and B. parapertussis (see table 3 ). For instance, only 4 (\#1, 3, 4 and 6) out of the 9 loci from $B$. pertussis, B. bronchiseptica or $B$. parapertussis show conservation of the genetic context in the 3 genomes (table 3). None of the genetic contexts is conserved in the B. avium genome (loci \#10 to 13$)$.

For some loci the numbers of tandem repeats vary between the different genomes. For example, locus \#3 contains 2 repeats in B. pertussis (between BP1793 and BP1794, lexA), 4 in B. parapertussis (between
BPP2022 and BPP2023, lexA) and 3 in B. bronchiseptica (between BB2270 and BB2271, lexA) (table 3, Figure 5). Locus \#2 is conserved only in B. pertussis and $B$. bronchiseptica. It has 2 repeats in $B$. pertussis (between BP1110, sphB3, and BP1111) and 8 in $B$. bronchiseptica (between BB2301, sphB3, and BB2302). Two out of 8 loci in B. bronchiseptica (loci \#7 and 8) have no homologous locus in the other genomes, and 1 out of 5 loci in the B. parapertussis genome (locus 9) has no homologous locus in the other genomes. A multiple alignment analysis on the Bordetella repeats shows a high level of conservation within the genus, as well as more widely within the all Betaproteobacteria class (Figure 6).

Several small repeats have been documented over the years in other prokaryotic species, including ERICs in enterobacteria [63-65], NEMIS in Neisseria spp. [66-68], the BOX and RUP elements in Streptococcus pneumoniae[69-71], MIRUs in Mycobacterium tuberculosis[72] and the bcr1 element of Bacillus cereus $[73,74]$. These repeats often feature characteristics of mobile elements and have a role in mRNA stabilization, transcription termination or promoter activity. BRE, described for the first time in this study, needs further investigation before we can suggest any potential function. Its heterogeneous chromosomal distribution and the fact that it is sometimes found in multiple copies at a same locus might imply a mobility or a past-mobility property. A preliminary search for BRE in sequences of other strains of B. pertussis, $B$. bronchiseptica and B. parapertussis revealed the presence of variable numbers of the repeat at the same locus among different strains (data not shown). This qualifies BREs as Variable Number Tandem Repeats (VNTR). This feature was verified using a VNTR analysis program [75], and most of identified loci were correctly predicted as VNTR, except for locus 3 in $B$. pertussis and B. bronchiseptica, and for locus 4 in $B$. bronchiseptica (data not shown). Additional investigations are under progress to assess BRE as potential genotyping markers.

\section{Conclusion}

$B$. pertussis and B. parapertussis, the second agent of whooping cough-like disease, have evolved relatively recently from a $B$. bronchiseptica-like ancestor, but these species have remained clonal, with a very limited number of $B$. pertussis-specific genes [76,77]. It is established that $B$. pertussis evolution and adaptation to humans occurred mainly through gene loss and recombination of the chromosome rather than through acquisition of new genes that would promote human 


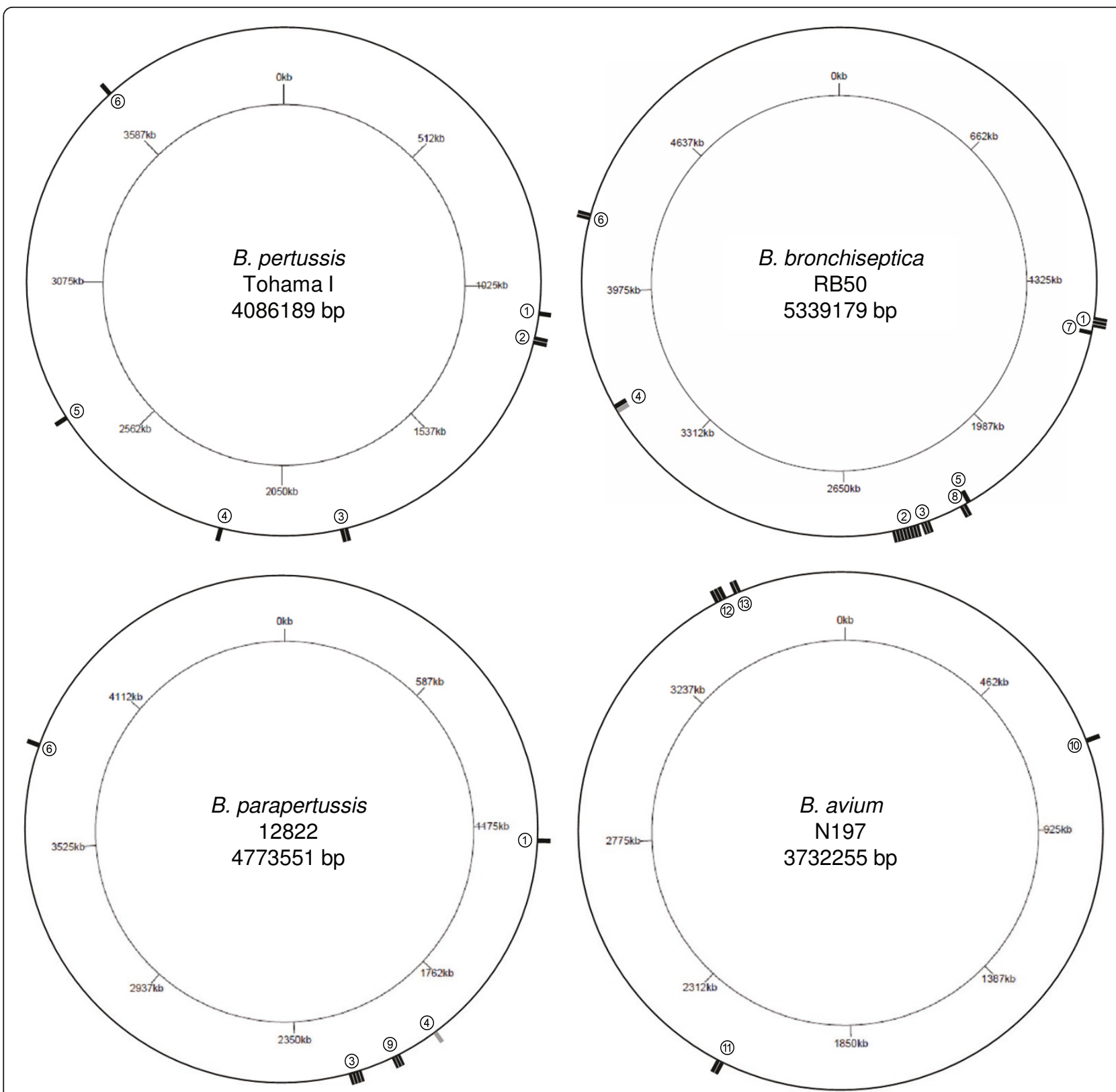

Figure 5 Distribution of BRE throughout the genomes of B. pertussis, B. bronchiseptica, B. parapertussis and B. avium. Repeats in the positive orientation (on the strand given in the GenBank genome database) are represented by a tick on the outside of the outer circles, and those in the negative orientation (on the complementary strand) are represented by a tick on the inside of the outer circles. The ticks in black are full-length repeats and ticks in grey are partial-length repeats. The number of ticks at each locus corresponds to the numbers of repeats. The inner circles give a chromosome graduation in kbp. Numbers on the outer circle indicate the locus number as defined in table 4.

infection [34]. It has also been suggested that certain genes are expressed differently in $B$. pertussis and in $B$. bronchiseptica, and that this difference might be responsible in part for infection in humans [78]. Such differences in expression may rely on classical base substitutions or indels in promoter regions of these genes but perhaps also on the specific action of unidentified regulatory molecules, such as sRNAs. In this study we have shown that $B$. pertussis, like other pathogens, expresses sRNAs and that the expression of one of them, BprJ2, is under the control of the BvgA/BvgS system. This sRNA and others, identified in this study or yet to be identified, could be important regulators for $B$. pertussis virulence acting either under the control of $\mathrm{BvgA} / \mathrm{BvgS}$ as co-regulators or as independent virulence regulators. 
Table 3 BRE loci genetic context in Bordetella genomes

\begin{tabular}{|c|c|c|c|c|c|c|c|c|c|c|c|c|c|c|c|c|c|c|c|c|}
\hline \multirow[b]{2}{*}{ Locus \# } & \multicolumn{5}{|c|}{ B. pertussis genetic contexte } & \multicolumn{5}{|c|}{ B. bronchiseptica genetic contexte } & \multicolumn{5}{|c|}{ B. pararpertussis genetic contexte } & \multicolumn{5}{|c|}{ B. avium genetic contexte } \\
\hline & Rpts. nbr. ${ }^{a}$ & $5^{\prime}$ gene & Std. ${ }^{\mathrm{b}}$ & 3' gene & Std. $^{b}$ & Rpts. nbr. ${ }^{a}$ & $5^{\prime}$ gene & Std. $^{b}$ & 3' gene & Std. ${ }^{b}$ & Rpts. nbr. ${ }^{a}$ & $5^{\prime}$ gene & Std. ${ }^{b}$ & 3' gene & Std. $^{\mathrm{b}}$ & Rpts. nbr. & $5^{\prime}$ gene & Std. $^{\mathrm{b}}$ & 3' gene & Std.b \\
\hline 1 & 1 & BP1052 & $>$ & BP1053 & $>$ & 3 & BB1365 & $>$ & BB1366 & $>$ & 1 & BPP1149 & $>$ & BPP1150 & $>$ & - & - & - & - & - \\
\hline 2 & 2 & BP1110 & $>$ & BP1111 & $>$ & 8 & BB2301 & $>$ & BB2302 & $>$ & - & - & - & - & - & - & - & - & - & - \\
\hline 3 & 2 & BP1793 & $>$ & BP1794 & $<$ & 3 & BB2270 & $>$ & BB2271 & $<$ & 4 & BPP2022 & $>$ & BPP2023 & $<$ & - & - & - & - & - \\
\hline 4 & 1 & BP2085 & $>$ & BP2086 & $>$ & 2 & BB3338 & $<$ & BB3339 & $<$ & 1 & BPP1769 & $>$ & BPP 1770 & $>$ & - & - & - & - & - \\
\hline 5 & 1 & BP2549 & $>$ & BP2550 & $<$ & 1 & BB2072 & $>$ & BB2073 & $<$ & - & - & - & - & - & - & - & - & - & - \\
\hline 6 & 1 & BP3395 & $<$ & BP3396 & $<$ & 2 & BB3983 & $<$ & BB3984 & $<$ & 1 & ВРP3548 & $<$ & ВРР3549 & $<$ & - & - & - & - & - \\
\hline 7 & - & - & - & - & - & 1 & BB1410 & $>$ & BB1411 & $>$ & - & - & - & - & - & - & - & - & - & - \\
\hline 8 & - & - & - & - & - & 2 & BB2091 & $>$ & BB2092 & $>$ & - & - & - & - & - & - & - & - & - & - \\
\hline 9 & - & - & - & - & - & - & - & - & - & - & 3 & BPP1903 & $>$ & BPP1904 & $>$ & - & - & - & - & - \\
\hline 10 & - & - & - & - & - & - & - & - & - & - & - & - & - & - & - & 1 & BAV0696 & $>$ & BAV0697 & $>$ \\
\hline 11 & - & - & - & - & - & - & - & - & - & - & - & - & - & - & - & 2 & BAV2006 & $>$ & BAV2007 & $>$ \\
\hline 12 & - & - & - & - & - & - & - & - & - & - & - & - & - & - & - & 3 & BAV3179 & $>$ & BAV3180 & $<$ \\
\hline 13 & - & - & - & - & - & - & - & - & - & - & - & - & - & - & - & 2 & BAV3208 & $>$ & BAV3209 & $<$ \\
\hline
\end{tabular}

'Rpts. nbr.' = Number of Repeats. Number of repeated BRE elements at the specific locus.

${ }^{b}$ 'Std.' = Strand. Genomic orientation of flanking mRNAs. ' $>$ ' = on positive strand: the strand given in the GenBank genome database; '<' = on negative strand: the complementary strand. 


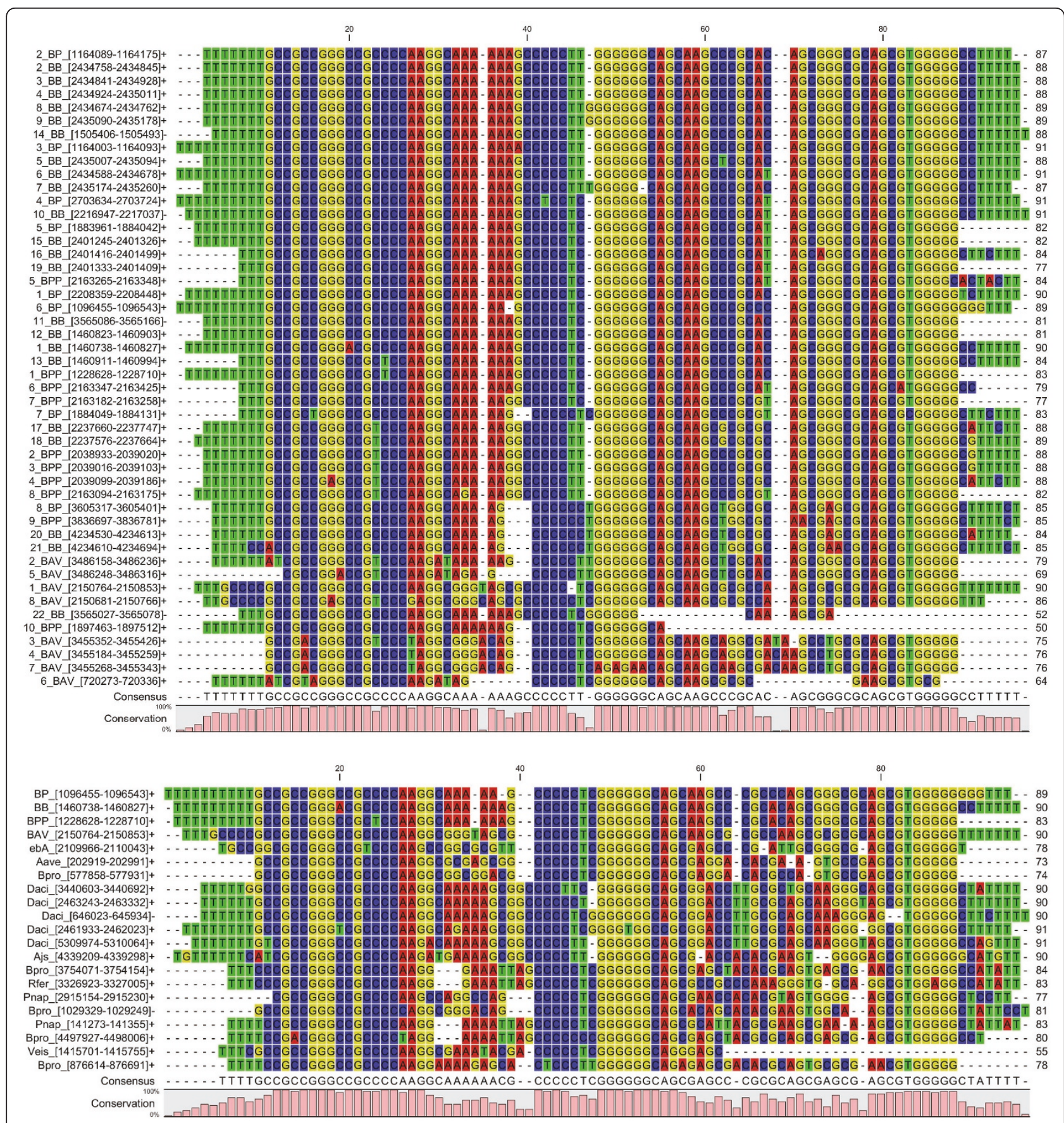

Figure 6 Multiple-alignment of Bordetella and betaproteobacteria BREs. The sequences were aligned using CLC Genomics Workbench 4. The name code of the bacterial strain and the repeat coordinates on the genomes are indicated on the left. Indels are represented by dashes. Upper panel: the 45 BREs from the 4 genomes B. pertussis, B. bronchiseptica, B. parapertussis, B. avium were aligned to evaluate conservation within the Bordetella genus. Lower panel: The BREs with the smallest coordinate in each betaproteobacteria genome were aligned to evaluate conservation within the all classes. 


\section{Additional material}

Additional file 1: Additional figure S1 - Additional tables S1, S2, S4

- Additional list S1. Figure S1: Preliminary Northern blot analysis.

Detection of transcripts in early(E), exponential(Ex) and stationary(S)

phases. Table S1: Biotin probe sequences used in northern blot analysis.

Table S2: RNAz prediction details. Table S4: BRE details. List S1: Bordetelle BRE sequences and positions.

Additional file 2: Additional figure S2 - Additional table S3. Figure

S2: Test for independent transcription between the putative sRNA

positions and the upstream or downstream ORF and RT-PCR primers.

Table S3: List of primers used in PCR described in figure S2.

\section{Acknowledgements}

This work was supported by PPF Bioinformatics Lille, the Institut Pasteur de Lille and the Institut National de la Santé et de la Recherche Médicale (INSERM).

We thank Julien Herrou (Inserm U629) for help with the bacterial culture and Hélène Touzet (INRIA, Lille) for help and discussion about bioinformatics studies.

\section{Author details}

${ }^{1}$ Center for Infection and Immunity of Lille (CIIL), Institut Pasteur de Lille, Lille, France. ${ }^{2}$ U1019, Institut National de la Santé et de la Recherche Médicale, Lille, France. ${ }^{3}$ UMR8204, Centre National de la Recherche Scientifique, Lille, France. ${ }^{4}$ Univ Lille Nord de France, Lille, France. ${ }^{5}$ IFR142, Molecular and Cellular Medicine, Lille, France. ${ }^{6}$ Genoscreen, Campus Institut Pasteur de Lille, Lille, France.

\section{Authors' contributions}

$\mathrm{DH}$ carried out the planning, design, data analysis and direction of the project, and he drafted the manuscript. SS carried out experimental work. $\mathrm{BW}, \mathrm{AD}$ and $\mathrm{CH}$ carried out bioinformatics and computational work. RA contributed to data analysis and interpretation and to the manuscript draft. $\mathrm{CL}$ and $\mathrm{YL}$ contributed to the planning and design of the project and to the manuscript. All authors read and approved the final manuscript.

\section{Competing interests}

The authors declare that they have no competing interests

Received: 14 December 2010 Accepted: 27 April 2011

Published: 27 April 2011

\section{References}

1. Dennis PP, Omer A: Small non-coding RNAs in Archaea. Curr Opin Microbiol 2005, 8(6):685-694.

2. Gottesman S: Micros for microbes: non-coding regulatory RNAs in bacteria. Trends Genet 2005, 21(7):399-404

3. Livny J, Waldor MK: Identification of small RNAs in diverse bacterial species. Curr Opin Microbiol 2007, 10(2):96-101.

4. Waters LS, Storz G: Regulatory RNAs in bacteria. Cell 2009, 136(4):615-628.

5. Gottesman S: The small RNA regulators of Escherichia coli: roles and mechanisms. Annu Rev Microbiol 2004, 58:303-328.

6. Moller T, Franch T, Hojrup P, Keene DR, Bachinger HP, Brennan RG, ValentinHansen P: Hfq: a bacterial Sm-like protein that mediates RNA-RNA interaction. Mol Cell 2002, 9(1):23-30.

7. Storz G, Opdyke JA, Zhang A: Controlling mRNA stability and translation with small, noncoding RNAs. Curr Opin Microbiol 2004, 7(2):140-144.

8. Babitzke P, Romeo T: CsrB sRNA family: sequestration of RNA-binding regulatory proteins. Curr Opin Microbiol 2007, 10(2):156-163.

9. Wassarman KM: 6S RNA: a small RNA regulator of transcription. Curr Opin Microbiol 2007, 10(2):164-168

10. Toledo-Arana A, Repoila F, Cossart P: Small noncoding RNAs controlling pathogenesis. Curr Opin Microbiol 2007, 10(2):182-188.

11. Boisset $S$, Geissmann $T$, Huntzinger $E$, Fechter $P$, Bendridi N, Possedko M, Chevalier C, Helfer AC, Benito Y, Jacquier A, et al: Staphylococcus aureus RNAIII coordinately represses the synthesis of virulence factors and the transcription regulator Rot by an antisense mechanism. Genes Dev 2007, 21(11):1353-1366.

12. Heroven AK, Bohme K, Rohde M, Dersch P: A Csr-type regulatory system, including small non-coding RNAs, regulates the global virulence regulator RovA of Yersinia pseudotuberculosis through RovM. Mol Microbiol 2008, 68(5):1179-1195

13. Johansson J, Mandin P, Renzoni A, Chiaruttini C, Springer M, Cossart P: An RNA thermosensor controls expression of virulence genes in Listeria monocytogenes. Cell 2002, 110(5):551-561.

14. Murphy ER, Payne SM: RyhB, an iron-responsive small RNA molecule, regulates Shigella dysenteriae virulence. Infect Immun 2007, 75(7):3470-3477

15. Padalon-Brauch G, Hershberg R, Elgrably-Weiss M, Baruch K, Rosenshine I, Margalit $\mathrm{H}$, Altuvia S: Small RNAs encoded within genetic islands of Salmonella typhimurium show host-induced expression and role in virulence. Nucleic Acids Res 2008, 36(6):1913-1927.

16. Romby P, Vandenesch F, Wagner EG: The role of RNAs in the regulation of virulence-gene expression. Curr Opin Microbiol 2006, 9(2):229-236.

17. Novick RP, Ross HF, Projan SJ, Kornblum J, Kreiswirth B, Moghazeh S: Synthesis of staphylococcal virulence factors is controlled by a regulatory RNA molecule. Embo J 1993, 12(10):3967-3975.

18. WHO: Pertussis vaccines-WHO position paper. Wkly Epidemiol Rec 2005, 80(4):31-39.

19. Crowcroft NS, Pebody RG: Recent developments in pertussis. Lancet 2006, 367(9526):1926-1936.

20. de Melker HE, Schellekens JF, Neppelenbroek SE, Mooi FR, Rumke HC, Conyn-van Spaendonck MA: Reemergence of pertussis in the highly vaccinated population of the Netherlands: observations on surveillance data. Emerg Infect Dis 2000, 6(4):348-357.

21. Guris D, Strebel PM, Bardenheier B, Brennan M, Tachdjian R, Finch E, Wharton M, Livengood JR: Changing epidemiology of pertussis in the United States: increasing reported incidence among adolescents and adults, 1990-1996. Clin Infect Dis 1999, 28(6):1230-1237.

22. Mooi FR, van Loo IH, King AJ: Adaptation of Bordetella pertussis to vaccination: a cause for its reemergence? Emerg Infect Dis 2001, 7(3 Suppl):526-528.

23. Cummings CA, Bootsma HJ, Relman DA, Miller JF: Speciesand strainspecific control of a complex, flexible regulon by Bordetella BvgAS J Bacteriol 2006, 188(5):1775-1785.

24. Hot $D$, Antoine $R$, Renauld-Mongenie $G$, Caro V, Hennuy B, Levillain E, Huot L, Wittmann G, Poncet D, Jacob-Dubuisson F, et al: Differential modulation of Bordetella pertussis virulence genes as evidenced by DNA microarray analysis. Mol Genet Genomics 2003, 269(4):475-486.

25. Jones AM, Boucher PE, Williams CL, Stibitz S, Cotter PA: Role of BvgA phosphorylation and DNA binding affinity in control of Bvg-mediated phenotypic phase transition in Bordetella pertussis. Mol Microbiol 2005, 58(3):700-713

26. Stibitz S: The bvg regulon. In Bordetella Molecular Microbiology. Edited by: Locht C. Norfolk (UK): Horizon Bioscience; 2007:

27. Prugnola A, Arico B, Manetti R, Rappuoli R, Scarlato V: Response of the bvg regulon of Bordetella pertussis to different temperatures and short-term temperature shifts. Microbiology 1995, 141(Pt 10):2529-2534.

28. Scarlato V, Arico B, Prugnola A, Rappuoli R: Sequential activation and environmental regulation of virulence genes in Bordetella pertussis. Embo J 1991, 10(12):3971-3975.

29. Trotochaud AE, Wassarman KM: A highly conserved 6S RNA structure is required for regulation of transcription. Nat Struct Mol Biol 2005, 12(4):313-319.

30. Felden B, Massire C, Westhof E, Atkins JF, Gesteland RF: Phylogenetic analysis of tmRNA genes within a bacterial subgroup reveals a specific structural signature. Nucleic Acids Res 2001, 29(7):1602-1607.

31. Scarlato V, Prugnola A, Arico B, Rappuoli R: Positive transcriptional feedback at the bvg locus controls expression of virulence factors in Bordetella pertussis. Proc Natl Acad Sci USA 1990, 87(17):6753-6757.

32. Scarlato V, Rappuoli R: Differential response of the bvg virulence regulon of Bordetella pertussis to MgSO4 modulation. J Bacteriol 1991 173(22):7401-7404

33. Washietl S, Hofacker IL, Stadler PF: Fast and reliable prediction of noncoding RNAs. Proc Natl Acad Sci USA 2005, 102(7):2454-2459.

34. Parkhill J, Sebaihia M, Preston A, Murphy LD, Thomson N, Harris DE, Holden MT, Churcher CM, Bentley SD, Mungall K, et al: Comparative 
analysis of the genome sequences of Bordetella pertussis, Bordetella parapertussis and Bordetella bronchiseptica. Nat Genet 2003, 35(1):32-40.

35. Sebaihia M, Preston A, Maskell DJ, Kuzmiak H, Connell TD, King ND, Orndorff PE, Miyamoto DM, Thomson NR, Harris D, et al: Comparison of the genome sequence of the poultry pathogen Bordetella avium with those of B. bronchiseptica, B. pertussis, and B. parapertussis reveals extensive diversity in surface structures associated with host interaction. J Bacteriol 2006, 188(16):6002-6015.

36. Menozzi FD, Mutombo R, Renauld G, Gantiez C, Hannah JH, Leininger E, Brennan MJ, Locht C: Heparin-inhibitable lectin activity of the filamentous hemagglutinin adhesin of Bordetella pertussis. Infect Immun 1994, 62(3):769-778.

37. Antoine R, Alonso S, Raze D, Coutte L, Lesjean S, Willery E, Locht C, JacobDubuisson F: New virulence-activated and virulence-repressed genes identified by systematic gene inactivation and generation of transcriptional fusions in Bordetella pertussis. J Bacterio/ 2000 182(20):5902-5905

38. NCBI ftp - Genomes Bacteria. [ftp://ftp.ncbi.nih.gov/genomes/Bacteria/]

39. Wellcome Trust Sanger Institute - Bacterial genomes. [http://www.sanger. ac.uk/Projects/Micr.bes/].

40. RNAz - predicting structural noncoding RNAs. [http://www.tbi.univie.ac.at/ $\sim$ wash/RNAz/].

41. FastPCR. [http://www.biocenter.helsinki.fi/bi/Programs/fastpcr.htm]

42. Noe L, Kucherov G: YASS: enhancing the sensitivity of DNA similarity search. Nucleic Acids Res 2005, 33 Web Server: W540-543.

43. YASS: DNA pairwise alignment tool.

44. Wellcome Trust Sanger Institute - Rfam. [http://rfam.sanger.ac.uk/]

45. Mandal M, Lee M, Barrick JE, Weinberg Z, Emilsson GM, Ruzzo WL, Breaker RR: A glycine-dependent riboswitch that uses cooperative binding to control gene expression. Science 2004, 306(5694):275-279.

46. Winkler WC, Cohen-Chalamish S, Breaker RR: An mRNA structure that controls gene expression by binding FMN. Proc Natl Acad Sci USA 2002, 99(25):15908-15913.

47. Livny J, Brencic A, Lory S, Waldor MK: Identification of 17 Pseudomonas aeruginosa sRNAs and prediction of sRNA-encoding genes in 10 diverse pathogens using the bioinformatic tool sRNAPredict2. Nucleic Acids Res 2006, 34(12):3484-3493

48. Vogel J, Argaman L, Wagner EG, Altuvia S: The small RNA IstR inhibits synthesis of an SOS-induced toxic peptide. Curr Biol 2004, 14(24):2271-2276.

49. Grundy FJ, Henkin TM: From ribosome to riboswitch: control of gene expression in bacteria by RNA structural rearrangements. Crit Rev Biochem Mol Biol 2006, 41(6):329-338.

50. Nudler $E$, Mironov AS: The riboswitch control of bacterial metabolism. Trends Biochem Sci 2004, 29(1):11-17.

51. Kaczanowska M, Ryden-Aulin M: Ribosome biogenesis and the translation process in Escherichia coli. Microbiol Mol Biol Rev 2007, 71(3):477-494.

52. Meyer MM, Ames TD, Smith DP, Weinberg Z, Schwalbach MS, Giovannoni SJ, Breaker RR: Identification of candidate structured RNAs in the marine organism 'Candidatus Pelagibacter ubique'. BMC Genomics 2009, 10:268

53. Lindahl $L$, Archer $R$, Zengel JM: Transcription of the $S 10$ ribosomal protein operon is regulated by an attenuator in the leader. Cell 1983, 33(1):241-248

54. Zengel JM, Mueckl D, Lindahl L: Protein L4 of the E. coli ribosome regulates an eleven gene $r$ protein operon. Cell 1980, 21(2):523-535.

55. NCBI - ORF Finder (Open Reading Frame Finder). [http://www.ncbi.nlm. nih.gov/gorf/gorf.html].

56. Nielsen $\mathrm{P}$, Krogh A: Large-scale prokaryotic gene prediction and comparison to genome annotation. Bioinformatics 2005, 21(24):4322-4329.

57. Hershberg R, Altuvia S, Margalit H: A survey of small RNA-encoding genes in Escherichia coli. Nucleic Acids Res 2003, 31(7):1813-1820.

58. Tjaden B: TargetRNA: a tool for predicting targets of small RNA action in bacteria. Nucleic Acids Res 2008, , 36 Web Server: W109-113.

59. Diwa A, Bricker AL, Jain C, Belasco JG: An evolutionarily conserved RNA stem-loop functions as a sensor that directs feedback regulation of RNase E gene expression. Genes Dev 2000, 14(10):1249-1260.

60. Melton AR, Weiss AA: Environmental regulation of expression of virulence determinants in Bordetella pertussis. J Bacterio/ 1989, 171(11):6206-6212.
61. Miller JF, Johnson SA, Black WJ, Beattie DT, Mekalanos JJ, Falkow S: Constitutive sensory transduction mutations in the Bordetella pertussis bvgS gene. J Bacteriol 1992, 174(3):970-979.

62. Knapp S, Mekalanos JJ: Two trans-acting regulatory genes (vir and mod) control antigenic modulation in Bordetella pertussis. J Bacteriol 1988, 170(11):5059-5066

63. Hulton CS, Higgins CF, Sharp PM: ERIC sequences: a novel family of repetitive elements in the genomes of Escherichia coli, Salmonella typhimurium and other enterobacteria. Mol Microbiol 1991, 5(4):825-834

64. Sharples GJ, Lloyd RG: A novel repeated DNA sequence located in the intergenic regions of bacterial chromosomes. Nucleic Acids Res 1990, 18(22):6503-6508.

65. Stern MJ, Ames GF, Smith NH, Robinson EC, Higgins CF: Repetitive extragenic palindromic sequences: a major component of the bacterial genome. Cell 1984, 37(3):1015-1026.

66. Buisine N, Tang CM, Chalmers R: Transposon-like Correia elements: structure, distribution and genetic exchange between pathogenic Neisseria sp. FEBS Lett 2002, 522(1-3):52-58.

67. Correia FF, Inouye $\mathrm{S}$, Inouye M: A family of small repeated elements with some transposon-like properties in the genome of Neisseria gonorrhoeae. J Biol Chem 1988, 263(25):12194-12198.

68. Mazzone M, De Gregorio E, Lavitola A, Pagliarulo C, Alifano P, Di Nocera PP. Whole-genome organization and functional properties of miniature DNA insertion sequences conserved in pathogenic Neisseriae. Gene 2001, 278(1-2):211-222.

69. Knutsen E, Johnsborg O, Quentin Y, Claverys JP, Havarstein LS: BOX elements modulate gene expression in Streptococcus pneumoniae: impact on the fine-tuning of competence development. J Bacterio/ 2006, 188(23):8307-8312.

70. Martin B, Humbert O, Camara M, Guenzi E, Walker J, Mitchell T, Andrew P, Prudhomme M, Alloing G, Hakenbeck R, et al: A highly conserved repeated DNA element located in the chromosome of Streptococcus pneumoniae. Nucleic Acids Res 1992, 20(13):3479-3483.

71. Oggioni MR, Claverys JP: Repeated extragenic sequences in prokaryotic genomes: a proposal for the origin and dynamics of the RUP element in Streptococcus pneumoniae. Microbiology 1999, 145(Pt 10):2647-2653.

72. Supply P, Magdalena J, Himpens S, Locht C: Identification of novel intergenic repetitive units in a mycobacterial two-component system operon. Mol Microbiol 1997, 26(5):991-1003.

73. Klevan A, Tourasse NJ, Stabell FB, Kolsto AB, Okstad OA: Exploring the evolution of the Bacillus cereus group repeat element bcr 1 by comparative genome analysis of closely related strains. Microbiology 2007, 153(Pt 11):3894-3908.

74. Okstad OA, Tourasse NJ, Stabell FB, Sundfaer CK, Egge-Jacobsen W, Risoen PA, Read TD, Kolsto AB: The bcr1 DNA repeat element is specific to the Bacillus cereus group and exhibits mobile element characteristics. J Bacteriol 2004, 186(22):7714-7725.

75. Denoeud F, Vergnaud G: Identification of polymorphic tandem repeats by direct comparison of genome sequence from different bacterial strains: a web-based resource. BMC Bioinformatics 2004, 5:4.

76. Cummings CA, Brinig MM, Lepp PW, van de Pas S, Relman DA: Bordetella species are distinguished by patterns of substantial gene loss and host adaptation. J Bacteriol 2004, 186(5):1484-1492

77. Diavatopoulos DA, Cummings CA, Schouls LM, Brinig MM, Relman DA Mooi FR: Bordetella pertussis, the causative agent of whooping cough, evolved from a distinct, human-associated lineage of B. bronchiseptica. PLoS Pathog 2005, 1(4):e45.

78. Preston A: Bordetella pertussis: the intersection of genomics and pathobiology. Cmaj 2005, 173(1):55-62.

doi:10.1186/1471-2164-12-207

Cite this article as: Hot et al:: Detection of small RNAs in Bordetella pertussis and identification of a novel repeated genetic element. BMC Genomics 2011 12:207. 